\title{
Two-port hand-assisted laparoscopic surgery for the 2-stage treatment of a complete bowel obstruction by left colon cancer: A case report
}

\author{
MASAYA MUKAI $^{1}$, AKIRA TANAKA ${ }^{1}$, TAKAYUKI TAJIMA ${ }^{1}$, MAKI FUKASAWA ${ }^{1}$, TAKESHI YAMAGIWA ${ }^{1}$, \\ KEN-ICHI OKADA ${ }^{1}$, KAZUHIKO SATO ${ }^{1}$, KOUSUKE TOBITA ${ }^{1}$, YASUHISA OIDA ${ }^{2}$ and HIROYASU MAKUUCHI ${ }^{2}$ \\ ${ }^{1}$ Tokai University Hachioji Hospital, Department of Surgery, Ishikawa-cho 1838, Hachioji, Tokyo 192-0032; \\ ${ }^{2}$ Tokai University School of Medicine, Department of Surgery, Bohseidai, Isehara, Kanagawa 259-1193, Japan
}

Received November 13, 2007; Accepted January 3, 2008

\begin{abstract}
A 69-year-old woman presented to her local clinic with vomiting and abdominal distension. Since a bowel obstruction by left colon cancer was suspected due to a marked dilation of the transverse colon, she was referred to our hospital. On admission, an enema disclosed a complete obstruction at the splenic flexure of the colon. An emergency operation was performed, and a temporary loop colostomy was fashioned on the left side of the transverse colon within the range of resection for 2-stage radical surgery. On hospital day 16, a left hemicolectomy D2 was performed by 2-port hand-assisted laparoscopic surgery (2P-HALS) using the stoma as the hand access site, and the tumor was resected along with the removal of the stoma. After surgery, a slight wound infection occurred at the hand access site, but this healed with conservative management. On day 36 , she was discharged from hospital. The histological diagnosis was Type 2 circumferential well-differentiated adenocarcinoma with local peritoneal dissemination. Our experience suggests that 2-stage surgery combined with $2 \mathrm{P}$-HALS is applicable even to a large obstructing left colon cancer. This method is less invasive, safe and achieves excellent results, including a good cosmetic outcome.
\end{abstract}

\section{Introduction}

Bowel obstruction occurs in $8-30 \%$ of all colorectal cancer patients, and it is not rare for this cancer to present with a

Correspondence to: Dr Masaya Mukai, Tokai University Hachioji Hospital, Department of Surgery, Ishikawa-cho 1838, Hachioji, Tokyo 192-0032, Japan

E-mail: mukai.masaya@hachioji-hosp.tokai.ac.jp

Abbreviations: 2P-HALS, 2-port hand-assisted laparoscopic surgery; 3P-HALS, 3-port hand-assisted laparoscopic surgery

Key words: colorectal cancer, laparoscopic surgery, hand-assisted laparoscopic surgery, laparoscopy-assisted surgery, colonic obstruction, oncological emergency rupture of the colon $(1,2)$. If the obstruction occurs proximal to the sigmoid colon, such as in the descending or left half of the transverse colon, decompression via the anus is difficult, resulting in complicated manipulations during emergency surgery in many cases (3). We treat emergency patients with obstruction of the left colon by cancer by initially fashioning a temporary loop colostomy for decompression within the range of resection at a subsequent radical operation. After the patient has been stabilized, the radical resection of the tumor and removal of the stoma are performed simultaneously (4). This method has the advantage that there is only one site of anastomosis and no influence on the extent of curative resection. The incidence of complications due to closure of the stoma, such as anastomotic leakage and/or stenosis, is very low. In recent years, hand-assisted laparoscopic surgery (HALS) has been reported to be useful, because it makes up for the disadvantages of standard laparoscopic surgery, such as the lack of tactile sensation for the surgeons and the need to use 4-6 multiple ports $(5,6)$. At our department, 2-port HALS (2P-HALS) with a small incision of 55-60 mm for hand access is used as the standard technique for laparoscopic surgery in patients with colon cancer. Herein, we report on a patient who underwent 2-stage surgery for obstructive colon cancer at the splenic flexure. Favorable results were obtained by fashioning an emergency loop colostomy and subsequent definitive surgery using 2P-HALS.

\section{Case report}

Onset and course. The patient was a 69-year-old woman who presented to her local clinic with severe constipation that had continued for several months, as well as nausea, vomiting and abdominal distension since around the end of July 2007. Plain abdominal X-ray films showed a marked large bowel dilation from the ascending to the transverse colon. On August 1, she was urgently referred to our hospital.

Past history. After enquiring into the past history of the patient, nothing contributory was found.

Laboratory tests. The results of the tests carried out showed WBC to be $9,400 / \mu 1$; RBC, $4.79 \times 10^{6} / \mu 1$; Hct, $42 \%$; Hb, $14 \mathrm{dl} / \mathrm{g}$; 


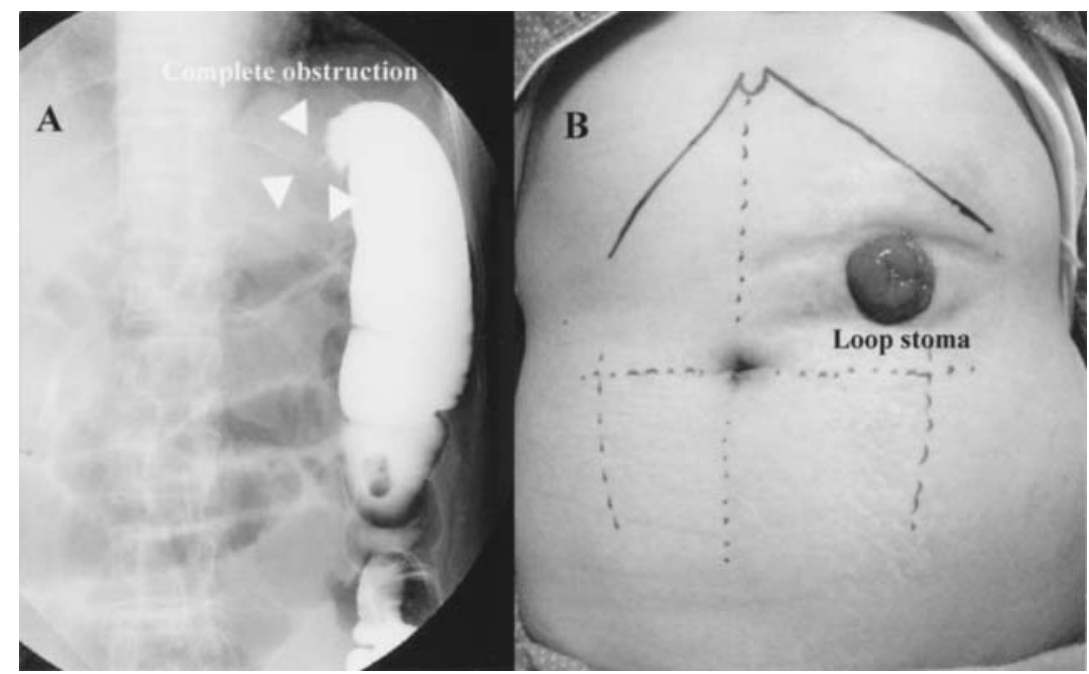

Figure 1. An emergency gastrografin enema at the first hospital visit. There is a complete obstruction at the splenic flexure of the transverse colon (arrow heads), and the oral part is not visualized at all (A). During emergency surgery, a loop stoma (left upper abdomen) was made in the left transverse colon, which was within the range of resection at subsequent radical surgery $(\mathrm{B})$.
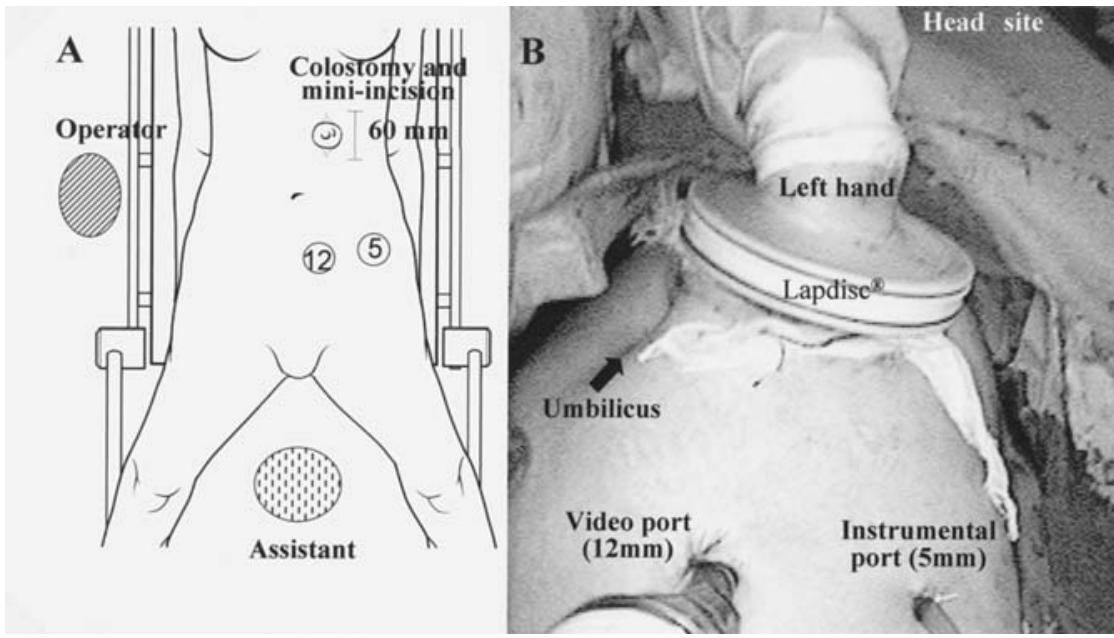

Figure 2. An illustration of the patient in the lithotomy position and the relationship between the ports and the operator/assistant (A). An overall view of the abdominal manipulation, including the left hand of the surgeon and the 2 ports (B).

platelet count, $2.58 \times 10^{5} / \mu \mathrm{l} ; \mathrm{CRP}, 1.12 \mathrm{mg} / \mathrm{dl} ; \mathrm{CEA}, 8.0 \mathrm{ng} / \mathrm{ml}$ and CA $19-9<1.0 \mathrm{U} / \mathrm{ml}$. Aside from a slight increase in the tumor markers, there were no abnormalities.

Imaging studies. An emergency enema with gastrografin showed a complete obstruction near the splenic flexure of the transverse colon (Fig. 1A) and a contrast CT scan disclosed an increasing tumor associated at the same site. Based on these findings, a complete obstruction of the left transverse colon by a tumor at the splenic flexure was diagnosed.

Emergency operation. On hospital day 1, an emergency operation was performed. A 35-mm skin incision was made to fashion a temporary loop colostomy on the left side of the transverse colon within the range of resection at subsequent radical surgery (Fig. 1B).
Elective $2 P$-HALS. On day 16, after intestinal decompression and improvement of her general condition, 2P-HALS was employed for radical tumor resection (Fig. 2A). The stoma was first dissected out and closed temporarily. Then it was reduced into the peritoneal cavity, and the skin incision at the stoma site was extended to $60 \mathrm{~mm}$ in length. A hand access device (Lapdisc ${ }^{\circledR}$, regular type, 120x120 mm, Hakkou Co. Ltd., Nagano, Japan) was fitted to this small incision, through which the left hand of the operator was inserted into the peritoneal cavity. Using the fingers as a guide, 2 ports (a 12-mm port for the video camera and a 5 -mm port for electric knives and forceps) were created by inserting trocars into the left lower abdomen (Fig. 2B). While retracting the colon with the left hand, the regions from the sigmoid colon to the splenic flexure with splenocolic ligament were mobilized by laparoscopy (Fig. 3A and B). Then the left colic artery and 


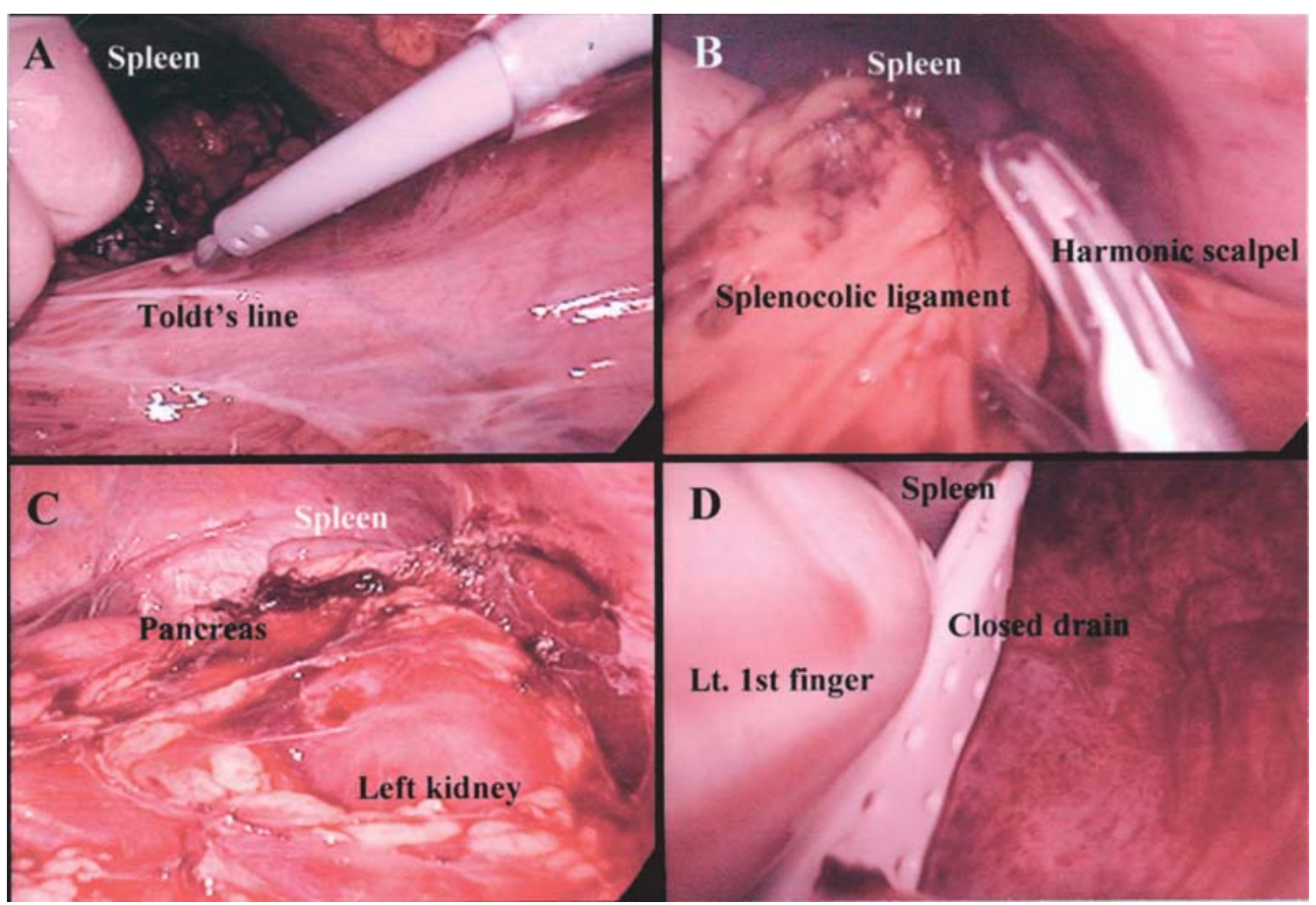

Figure 3. The large bowel is manipulated with the fingers, and the sigmoid colon is mobilized by the external approach (A). An intra-abdominal view, including the splenocolic ligament (B), the pancreatic body, tail and spleen, after D2 left hemicolectomy (C). After the insertion of a drain tube in the left subphrenic space (D).

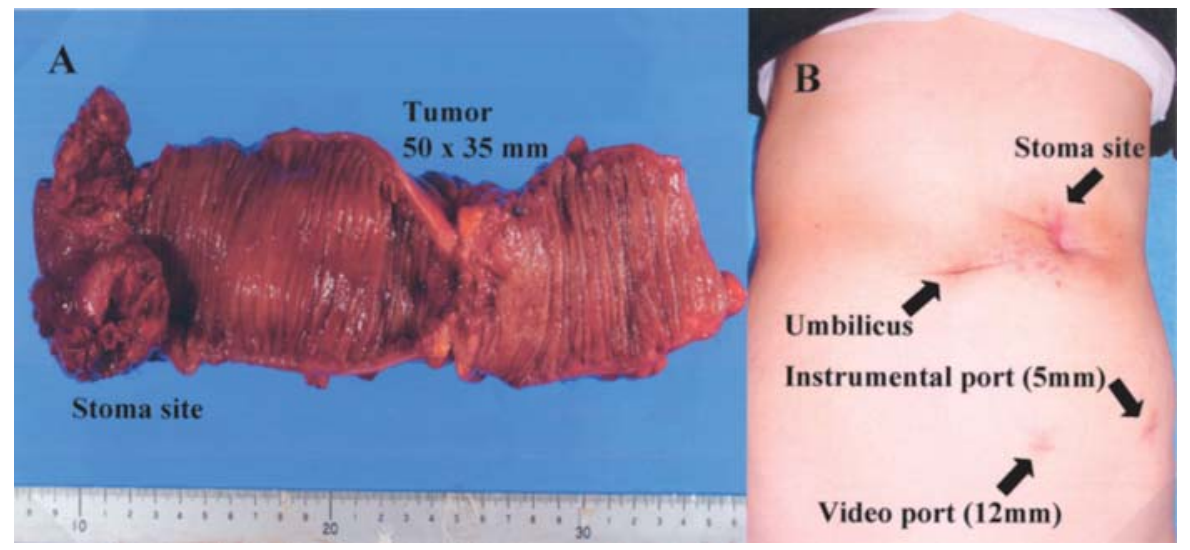

Figure 4. The stoma site (oral side) and the primary tumor $(50 \times 35 \mathrm{~mm})$ are removed adequately along with a radical D2 dissection (A). On day 60 , the wound infection at the old stoma site has completely healed to a small scar (B).

vein were ligated at the root and dissections of the transverse mesocolon were performed at the lower margin of the body and tail of the pancreas (Fig. 3C). The middle colic artery and vein were confirmed under direct vision through the small laparotomy wound. Although it was possible to perform D3 lymph node dissection under direct vision, since the disseminated peritoneal nodules were seen in the adjacent greater omentum (P1), D2 left hemicolectomy was performed after ligation of the left branch of the middle colic artery and vein. After the resected part of the transverse mesocolon was closed completely under direct vision, extra-abdominal functioning end-to-end anastomosis was performed. Then a pneumoperitoneum was created again, and surgery was completed by inserting a closed drain below the left subphrenic space (Fig. 3D). The operating time, including removal of the stoma, was $170 \mathrm{~min}$ and the blood loss was $84 \mathrm{ml}$.

Pathological diagnosis. The histological diagnosis was Type 2 circumferential $(50 \times 35 \mathrm{~mm})$ well-differentiated adenocarcinoma with localized peritoneal dissemination in the vicinity of the tumor. The final pathological diagnosis was $\mathrm{P} 1 \mathrm{p}(+) \mathrm{H} 0 \mathrm{pSSpN}_{2}(6 / 13) \mathrm{M}(-)$, Stage IV advanced transverse colon cancer (Fig. 4A).

Postoperative course. After surgery, a slight wound infection was noted at the site of the stoma closure, but it healed with conservative management. The patient was discharged from hospital on day 36 . On day 60 , the wound was completely 
healed and closed (Fig. 4B). At 3 months after surgery, the patient is receiving systemic chemotherapy on an outpatient basis.

\section{Discussion}

Since Jacobs et al in Florida reported 20 patients who underwent laparoscopy-assisted resection of the large bowel in 1991 (6), various new surgical techniques have been developed consecutively and the colorectal cancer patients for whom this surgery is indicated have rapidly increased in number. These techniques have been improved and modified in various ways at different institutions (7-10). In Japan, the most common surgical technique is an internal approach of laparoscopic surgery without hand assistance, in which blood vessels at the mesenteric root are pretreated using 4-6 ports including a camera port $(6-8,11)$. However, laparoscopic surgery is more time-consuming than standard laparotomy because it depends on visual information from a camera, and it is often contra-indicated because of the difficulty in manipulation when patients have advanced cancer with a relatively large diameter or tumors associated with adhesions and multiple organ involvement. On the other hand, more reports have recently been published concerning hybrid surgery, so-called HALS, in Europe and the USA (12-14). HALS has many advantages over current laparoscopic surgery, such as tactile sensation, palpation, strong traction and the use of fewer ports. If the latest hand access device is used, it is possible to create a pneumoperitoneum when the hand is not inserted. Inserting a camera and a trocar via the device and open surgical manipulation is also possible with direct vision and/or via laparoscopy as a hybrid HALS method. It has been reported that there are no differences between these surgical techniques and open laparotomy with respect to the number of dissected lymph nodes and the distance between the oral and anal stumps. It has also been reported by many researchers that it is easier to learn this method than the standard laparoscopic surgery (12-14).

At our department, 2P-HALS with D2 or D3 dissection and 3P-HALS with D2 dissection have been used since May 2007 for the colon and rectum, respectively, as the standard surgical techniques for colorectal cancer, and we have obtained satisfactory results with these methods in $\sim 20$ patients. For right or left hemicolectomy, a 55- to $60-\mathrm{mm}$ longitudinal median skin incision is made $\sim 4-5 \mathrm{~cm}$ below the xiphoid process. Then a hand access device is attached. A $12-\mathrm{mm}$ camera port and a $5-\mathrm{mm}$ port are placed in the right or left lower abdomen by the direct puncture method and are guided by the fingers of the left hand while the bladder is avoided. In patients with sigmoid colon or rectal cancer, a 55- to 60-mm transverse skin incision is made just above the pubis. After the peritoneal cavity has carefully been observed, the sigmoid colon is mobilized by the external approach. As a result, a satisfactory visual field for circumferential dissection and elevation can be obtained in the pelvic cavity, owing to sufficient direct traction by the left hand. Apart from the complicated dissection of the rectum at the pelvic floor, additional forceps are rarely needed for contralateral traction or the dissection of the splenocolic ligament and transverse mesocolon at the pancreatic body and tail. Only one forcep port is needed in most cases, including the present one. Since hybrid HALS is by no means inferior to the traditional median laparotomy with respect to visualizing the surgical field and dissection of layers, unlike standard laparoscopic surgery, it may be indicated for tumors of almost all sizes and weights.

Since the right colon is characterized by a large diameter and liquid contents, complete obstruction at this site is relatively rare. Even when bowel obstruction develops, decompression may be possible using a long ileus tube. Moreover, when emergency surgery is performed without preoperative bowel lavage, since the bowel contents are not fecal, it is relatively rare for surgeons to feel hesitation about performing anastomosis after one-stage resection of the tumor. Therefore, a complete obstruction of the left colon by cancer is a more serious problem (15-18). At initial surgery, the radical resection of the primary tumor usually involves the removal of the cancer, followed by bowel anastomosis plus a temporary loop ileostomy and/or right transverse loop colostomy (19-21). One-stage anastomotic reconstruction is also possible, in which massive bowel lavage is performed from the oral side during surgery for the purpose of avoiding a temporary stoma. However, these surgical procedures have some serious disadvantages because the operating time is relatively long and surgical invasion is too great for elderly patients undergoing emergency surgery (22-24). It has also been reported that these procedures may be associated with serious complications, such as the intraoperative rupture of a dilated large bowel, DIC, serious wound infection and dehiscence, suture failure and peritonitis $(25,26)$. Hartmann's operation is also available, in which anastomosis is not performed after the resection of the primary tumor, and the oral stump is used as a single-lumen stoma. However, when this technique is used, it is often difficult to remove intrapelvic adhesions and perform re-anastomosis, so it is not uncommon for re-anastomosis to be abandoned because of the high risk in elderly patients $(27,28)$.

It has been reported that temporary loop colostomy is the best initial emergency operation for the complete obstruction of the left colon by cancer, because it can be performed promptly at any time and it is less invasive while definitely achieving decompression (4). We created a loop colostomy for decompression that was not in the right transverse colon or ileum, but was located within the range of resection at subsequent radical surgery (4). As a result, elective surgery was performed safely after bowel dilation had resolved $\sim 2$ weeks later and the condition of the patient had improved sufficiently. The delay also allowed for the careful assessment of the risks involved. In this case, postoperative wound infection was considered to be unavoidable because of the hand access port being placed at the dirty stoma wound. However, management as a partly-open wound meant that no severe complications occurred, such as rectus muscle infection, peritonitis or abdominal wall dehiscence. A small wound infection was controlled by irrigation and dressings and the patient recovered fully. This patient could be treated safely and even the cosmetic outcome was satisfactory when the temporary loop stoma made during initial emergency surgery was utilized as the hand access site for 2P-HALS. It will be necessary to investigate the long-term outcome of hybrid 2P/3P-HALS in a larger number of patients in the future. 


\section{Acknowledgements}

This study was supported by grants from the Occult Neoplastic Cells Research and Study Group (\#2007-5007, Tokai University Hachioji Hospital, Tokyo, Japan) and the Research and Study Program of Tokai University Educational System General Research Organization (\#2007-04, Tokai University Hospital, Kanagawa, Japan).

\section{References}

1. Kronborg O, Backer O and Sprechler M: Acute obstruction in cancer of the colon and rectum. Dis Colon Rectum 18: 22-27, 1975 .

2. Stower MJ and Hardcastle JD: The results of 1115 patients with colorectal cancer treated over an 8-year period in a single hospital. Eur J Surg Oncol 11: 119-123, 1986.

3. Chen HS and Sheen-Chen SM: Obstruction and perforation in colorectal adenocarcinoma: an analysis of prognosis and current trends. Surgery 127: 370-376, 2000.

4. Mukai M, Himeno S, Mukoyama S, Tajima T, Saito Y, Ito I, Nakasaki H, Sato S and Makuuchi H: Is the temporally loop colostomy in the right transverse colon appropriate for complete obstruction by colorectal cancer? Oncol Rep 10: 693-698, 2003.

5. Anderson J, Luchtefeld M, Dujovny N, Hoedema R, Kim D and Butcher J: A comparison of laparoscopic, hand-assisted and open sigmoid resection in the treatment of diverticular disease. Am J Surg 193: 400-403, 2007.

6. Jacobs M, Verdja JC and Goldstein HS: Minimally invasive colon resection (laparoscopic colectomy). Surg Laparosc Endosc 1: 144-150, 1991 .

7. Mukai M, Sadahiro S, Tokunaga N, Ishida H, Makuuchi H, Tajima T and Mitomi T: Clinical experiences with laparoscopic colectomy. Dig Endosc 9: 11-15, 1997.

8. The clinical outcomes of surgical therapy study group. A comparison of laparoscopically assisted and open colectomy for colon cancer. N Engl J Med 350: 2050-2059, 2004.

9. Yamaguchi Y, Minami K, Kawabuchi Y, Emi M and Toge T: Anterior resection of rectal cancer through a one hand-size incision with or without laparoscopy: proposal of one hand-size incision surgery (OHaSIS). J Surg Res 129: 136-141, 2005.

10. Ichihara T, Takada M, Fukumoto S, Yasuda T and Kuroda Y: A novel technique of finger-assisted laparoscopic surgery. Am J Surg 187: 285-287, 2004.

11. Vithiananthan S, Cooper Z, Betten K, Stapleton GS, Carter J, Huang EH and Whelan RL: Hybrid laparoscopic flexure takedown and open procedure for rectal resection is associated with significantly shorter length of stay than equivalent open resection. Dis Colon Rectum 44: 927-935, 2001.
12. Romanelli JR, Kelly JJ and Litwin DE: Hand-assisted laparoscopic surgery in the United States: an overview. Semin Laparosc Surg 8: 96-103, 2001

13. Nakajima K, Lee SW, Cocilovo C, Foglia C, Kim K, Sonoda T and Milson JW: Hand-assisted laparoscopic colorectal surgery using GelPort. Surg Endosc 18: 102-105, 2004.

14. Nakajima K, Lee SW, Cocilovo C, Foglia C, Sonoda T and Milson JW: Laparoscopic total colectomy: hand-assisted vs. standard technique. Surg Endosc 18: 582-586, 2004.

15. Hoffmann J and Jensen HE: Tube cecostomy and staged resection for obstructing carcinoma of the left colon. Dis Colon Rectum 27: 24-32, 1984.

16. Mackenzie S, Thompson SR and Baker LW: Management options in malignant obstruction of the left colon. Surg Gynecol Obstet 74: 337-344, 1992.

17. Sjödahl R, Franzen T and Nyström PO: Primary versus staged resection for acute obstructing colorectal carcinoma. Br J Surg 79: 685-688, 1992.

18. Williams NS, Nasmyth DG, Jones D and Smith AH: Defunctioning stomas: a prospective controlled trial comparing loop ileostomy with loop transverse colostomy. Br J Surg 73: 566-570, 1986.

19. Gooszen AW, Geelkerken RH, Hermans J, Lagaay MB and Gooszen HG: Temporary decompression after colorectal surgery: randomized comparison of loop ileostomy and loop colostomy. Br J Surg 85: 76-79, 1998.

20. Tschmelitsch J, Wykypiel H, Prommegger R and Bodner E: Colostomy vs. tube cecostomy for protection of low anastomosis in rectal cancer. Arch Surg 134: 1385-1388, 1999.

21. Rullier E, Le Toux N, Laurent C, Garrelon J-L, Parneix M and Saric J: Loop ileostomy versus loop colostomy for defunctioning low anastomoses during rectal cancer surgery. World J Surg 25: 274-278, 2001.

22. Stothert JC Jr, Brubacher L and Simonowitz DA: Complications of emergency stoma formation. Arch Surg 120: 1145-1147, 1985.

23. Thomson WHF and Carter SS: On-table lavage to achieve safe restorative rectal and emergency left colonic resection without covering colostomy. Br J Surg 73: 61-63, 1986.

24. Pollock AV, Playforth MJ and Evans M: Peroperative lavage of the obstructed left colon to allow safe primary anastomosis. Dis Colon Rectum 30: 171-173, 1987.

25. Porter JA, Salvati EP, Rubin RJ and Eisentat TE: Complications of colostomies. Dis Colon Rectum 32: 299-303, 1989.

26. Karanjia ND, Corder AP, Holdsworth PJ and Heald RJ: Risk of peritonitis and fatal septicaemia and the need to defunction the low anastomosis. Br J Sur 78: 196-198, 1991.

27. Schein M and Decker G: The Hartmann procedure: extended indications in severe intra-abdominal infection. Dis Colon Rectum 31: 126-129, 1987.

28. Keck JM, Collopy BT, Ryan PJ, Fink R, Mackay JR and Woods RJ: Reversal of Hartmann's procedure: effect of timing and technique on ease and safety. Dis Colon Rectum 37: 243-248, 1994. 